\title{
Ärztestopp als Kostenbremse - Warum es Zeit ist umzudenken
}

\author{
B. Horisberger*, O. Schmid*
}

\section{Einleitung}

Gemäss dem Bundesamt für Statistik sind die Gesundheitsausgaben, gemessen am Bruttosozialprodukt (BIP), in den letzten 40 Jahren kontinuierlich von 4,9\% (1960) auf 10,7\% (2000) gestiegen [1]. Die Gesundheitskosten pro Kopf der Bevölkerung liegen in der Schweiz heute höher als in allen anderen europäischen Ländern [2].

Das öffentliche Interesse an den Kosten des Gesundheitswesens konzentriert sich hierzulande seit einigen Jahren insbesondere auf die Leistungen der Krankenversicherer und auf die Versicherungsprämien. Im Mittelpunkt der Debatten stehen dabei die Leistungen in der Grundversicherung. Davon betroffen sind in erster Linie die privaten Haushalte, welche insgesamt etwa zwei Drittel aller «Kosten des Gesundheitswesens» tragen und die Versicherungsleistungen über Mitgliederbeiträge und Kostenbeteiligungen finanzieren [3]. Zwischen 1999 und 2002 sind die Bruttoleistungen der KVG-Versicherer, pro Versicherte, insgesamt von Fr. 2014.- auf Fr. 2354.- pro Jahr angestiegen (+16,9\%), und die kantonale Durchschnittsprämie für Erwachsene erhöhte sich von Fr. 2448.- auf Fr. 2940.$(+20,1 \%)[4,5]$.

Viele Politiker sehen in der Zunahme der Leistungserbringer, vor allem in der freien Praxis (Stichwort Ärztedichte), die Hauptursache für die Kostenentwicklung im Gesundheitswesen. Sie werden in ihrer Annahme bestärkt durch die Tatsache, dass Kantone mit einer höheren Ärztedichte auch höhere Gesundheitskosten aufweisen (Abb. 1).

\footnotetext{
* Es bestehen keine Interessensverbindungen wie fremde finanzielle Mittel oder persönliche Verbindungen im Zusammenhang mit der eingereichten Arbeit.
}

Korrespondenz:

Dr. oec. Olivier Schmid

Institut für Unternehmensforschung Universität St. Gallen

Holzstrasse 15

CH-9010 St. Gallen

Tel. 0712242461

Fax 0712242102

E-Mail: olivier.schmid@unisg.ch

\section{Zulassungsbeschränkung}

\section{für neue Leistungserbringer}

Am 3. Juli 2002 hat der Bundesrat die Regeln einer bedürfnisabhängigen Zulassungsbeschränkung für neue Leistungserbringer zur Tätigkeit zu Lasten der obligatorischen Krankenpflegeversicherung festgelegt [6]. Im Anhang 1 zur Verordnung wird für jeden Kanton die maximale Anzahl Leistungserbringer für die verschiedenen ärztlichen Fachrichtungen und die anderen Gesundheitsberufe, die in der Grundversicherung abrechnen dürfen, als Richtwert vorgegeben. Dieser orientiert sich im wesentlichen am Status quo. Den Kantonen sollte damit ein Instrument in die Hand gegeben werden, um die Kostenentwicklung einzudämmen. Das BSV bezeichnete die Verordnung in einer Medienmitteilung als «Notbremse» [7].

Diese Massnahme entspricht der Vorstellung eines angebotsinduzierten Kostenverursachermodells. Die These, dass die Ärztedichte die Menge der Leistungen zu einem bestimmten Zeitpunkt bestimmt, stösst in der wissenschaftlichen Literatur auf breite Zustimmung [8-11]. Hingegen ist die Annahme, dass die Ärztedichte auch das Wachstum der Gesundheitskosten entscheidend beeinflusst, umstritten. Das Schrifttum über die Dynamik der Kostenentwicklung in Abhängigkeit von der Ärztedichte ist im Gegensatz zur Fülle der Publikationen über konsumfördernde Effekte spärlich. Längerfristig werden die Kostensteigerungen als Folge des medizinisch-technischen Fortschritts, der Einführung neuer und teurerer Medikamente, der Zunahme der ambulanten Spitalbehandlungen, der Nachfragesteigerung aus demographischen Gründen und der höheren Pflegeheimkosten für mindestens so wichtig gehalten wie die angebotsinduzierte Kostensteigerung durch die Ärzte [12].

Damit stellt sich die Schlüsselfrage, ob ein gesicherter Zusammenhang zwischen der Ärztedichte und dem Anstieg der Kassenleistungen überhaupt nachgewiesen werden kann. Der neue Aspekt dieser Arbeit ist die Analyse des prozentualen Kostenwachstums in der obligatorischen Krankenversicherung in Abhängigkeit von der Ärztedichte 1999 und der Veränderung derselben zwischen 1999 und 2002 in den 26 Kantonen.

\section{Material und Methode}

\section{Material}

Als Grundlage für unsere Berechnungen benützten wir für die Kostenermittlung den santésuisseDatenpool [4], für die Prämienerhebung die schweizerische Sozialversicherungsstatistik, herausgegeben vom Bundesamt für Sozialversicherung [5], und zur Erfassung der Ärztedichten die FMH-Ärztestatistik [13, 14]. Die verwendeten Daten werden in Tabelle 1 festgehalten. 


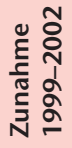

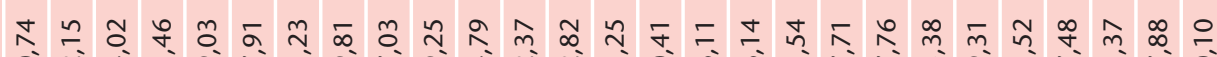

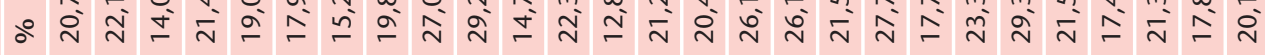

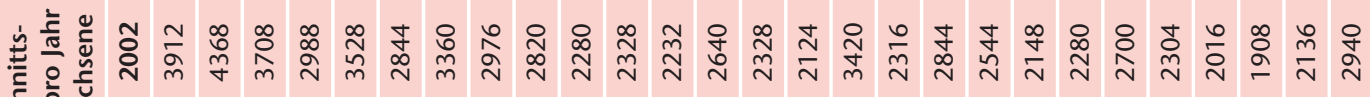
舵

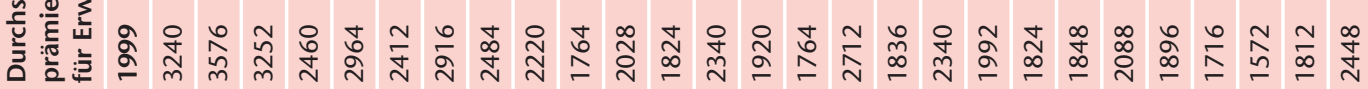

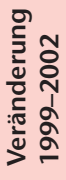

으

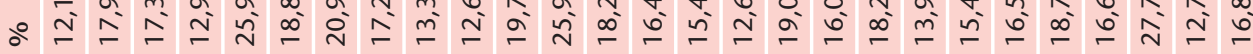

可

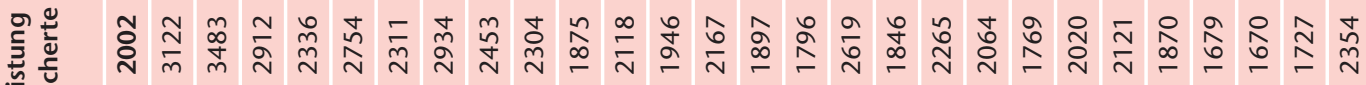

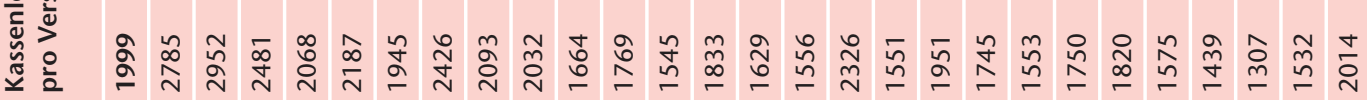

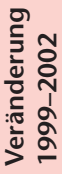

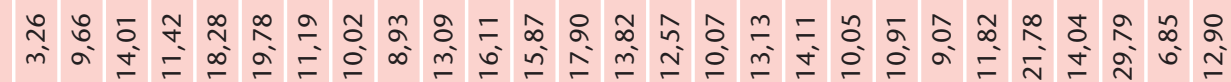

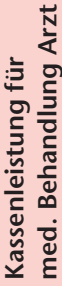

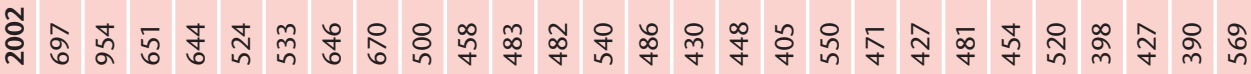

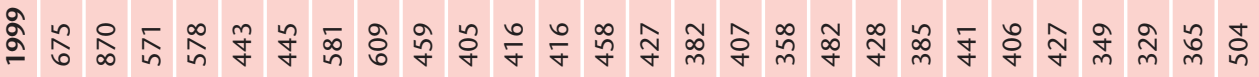

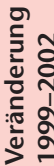

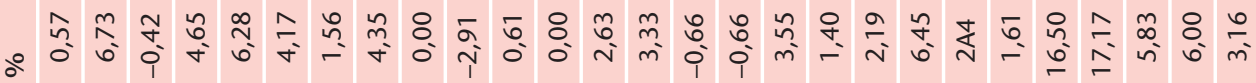

응

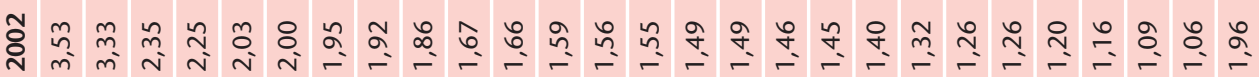
gे 
Abbildung 1

Ärztedichte und Kassenleistungen sowie Krankenversicherungsprämien 2002.

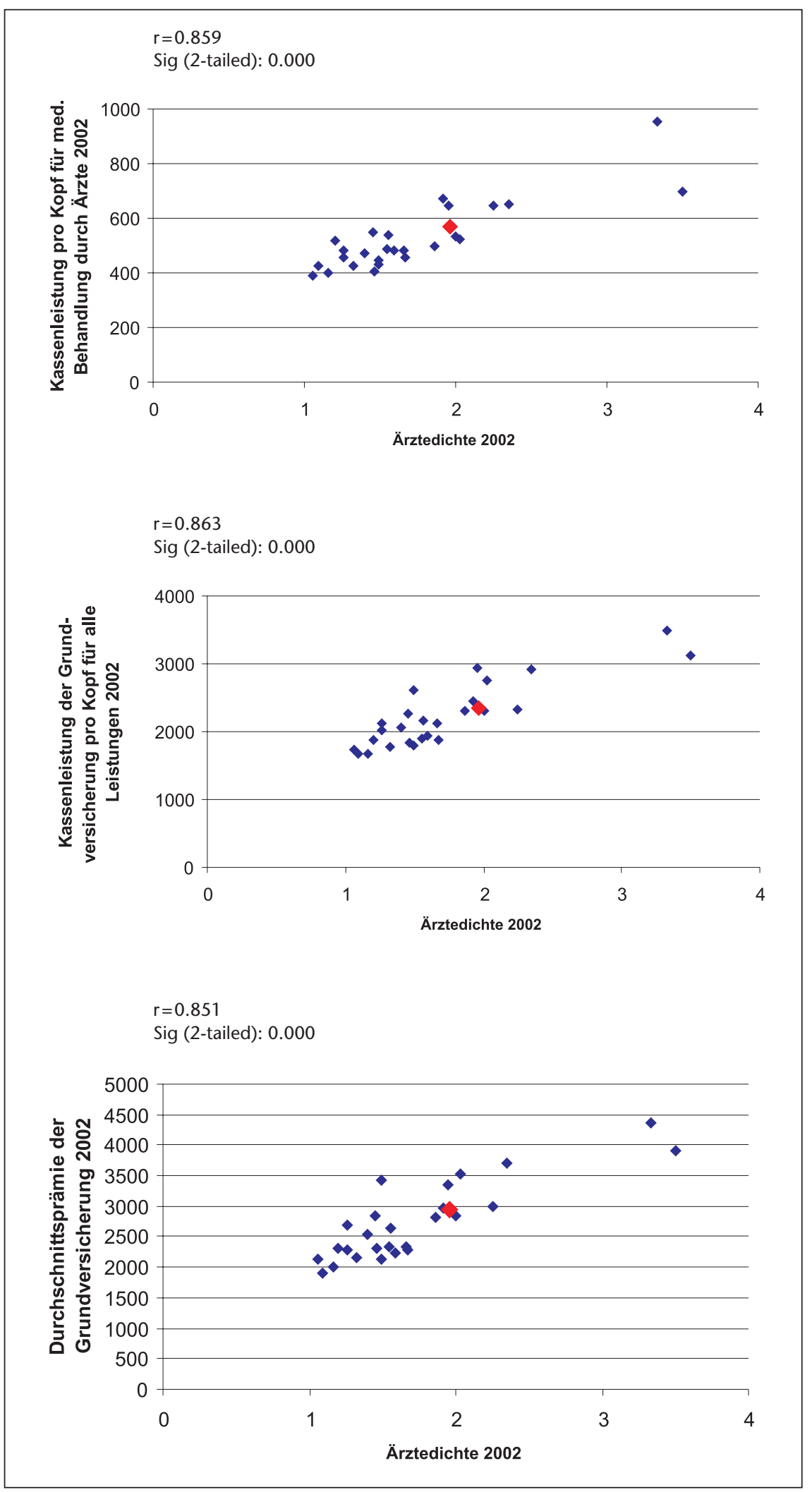

Tabelle 2

Versicherungsleistungen insgesamt pro Versicherte in 26 Kantonen 1999-2002.

\begin{tabular}{|c|c|c|c|c|}
\hline Kanton & 1999 & 2000 & 2001 & 2002 \\
\hline AG & 1745 & 1877 & 1981 & 2064 \\
\hline $\mathrm{Al}$ & 1307 & 1438 & 1539 & 1670 \\
\hline AR & 1556 & 1676 & 1748 & 1796 \\
\hline BE & 1945 & 2161 & 2224 & 2311 \\
\hline$B L$ & 2093 & 2224 & 2362 & 2453 \\
\hline BS & 2785 & 2927 & 3099 & 3122 \\
\hline FR & 1951 & 2020 & 2155 & 2265 \\
\hline GE & 2952 & 3085 & 3366 & 3483 \\
\hline GL & 1750 & 1875 & 1947 & 2020 \\
\hline GR & 1664 & 1838 & 1844 & 1875 \\
\hline JU & 2326 & 2352 & 2526 & 2619 \\
\hline LU & 1551 & 1668 & 1788 & 1846 \\
\hline NE & 2187 & 2589 & 2718 & 2754 \\
\hline NW & 1439 & 1601 & 1637 & 1679 \\
\hline OW & 1532 & 1651 & 1693 & 1727 \\
\hline SG & 1629 & 1716 & 1800 & 1897 \\
\hline $\mathrm{SH}$ & 2032 & 2140 & 2252 & 2304 \\
\hline SO & 1833 & 1949 & 2039 & 2167 \\
\hline SZ & 1575 & 1700 & 1876 & 1870 \\
\hline TG & 1820 & 1897 & 1973 & 2121 \\
\hline TI & 2426 & 2642 & 2768 & 2934 \\
\hline UR & 1553 & 1682 & 1850 & 1769 \\
\hline VD & 2481 & 2676 & 2852 & 2912 \\
\hline VS & 1769 & 1933 & 2031 & 2118 \\
\hline ZG & 1545 & 1664 & 1848 & 1946 \\
\hline ZH & 2068 & 2172 & 2277 & 2336 \\
\hline $\mathrm{CH}$ & 2014 & 2161 & 2272 & 2354 \\
\hline
\end{tabular}

\section{Methoden der Statistik}

Die Abhängigkeit zwischen der Ärztedichte und den Kassenleistungen bzw. den Versicherungsprämien wurde anhand der Pearson-Korrelation gemessen. Die Korrelationswerte wurden jeweils mittels t-Test auf Signifikanz geprüft. Sämtliche Berechnungen sind mit der Statistiksoftware SPSS vorgenommen worden.

Um den dynamischen Zusammenhang zwischen den Variablen zu messen, wurden die Korrelationen zwischen der Ärztedichte 1999 und den prozentualen Veränderungen der Kassenleistungen bzw. der Versicherungsprämien zwischen 1999 und 2002 analysiert. In einer zweiten Analyse wurde der Zusammenhang zwischen der prozentualen Veränderung der Ärztedichten und der prozentualen Veränderung der Kassenleistungen bzw. der Versicherungsprämien in den Jahren 1999-2002 ermittelt. 
Abbildung $2 \mathrm{a}-\mathrm{c}$

Prozentuale Veränderung der Kassenleistungen und Versicherungsprämien 1999-2002 in Beziehung zur Ärztedichte 1999 in 26 Kantonen.

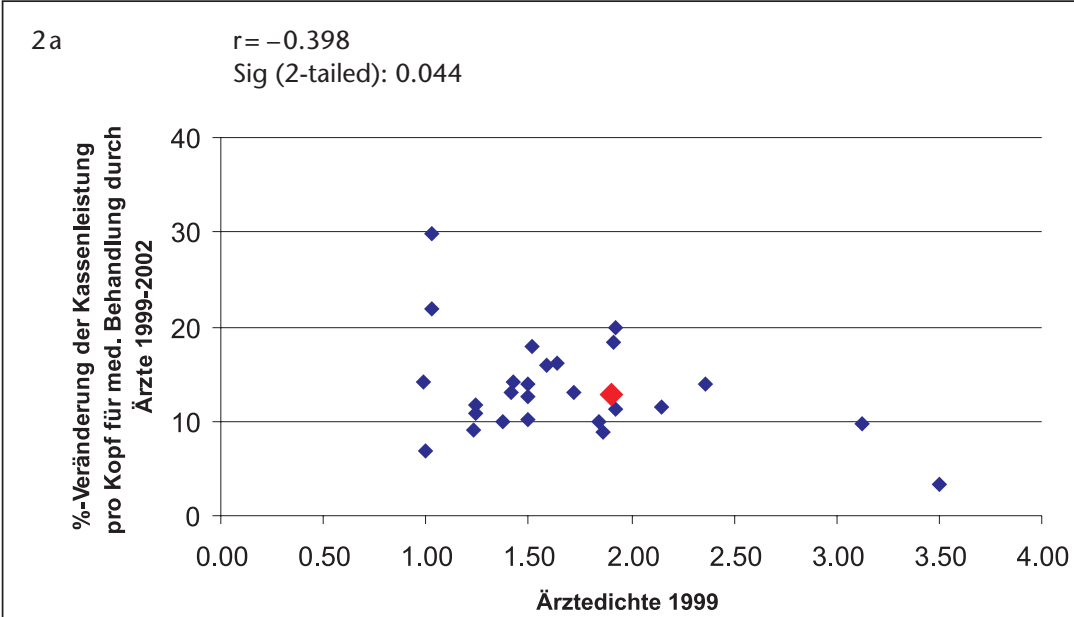

$2 \mathrm{~b}$

$r=-0.157$

Sig (2-tailed): 0.445

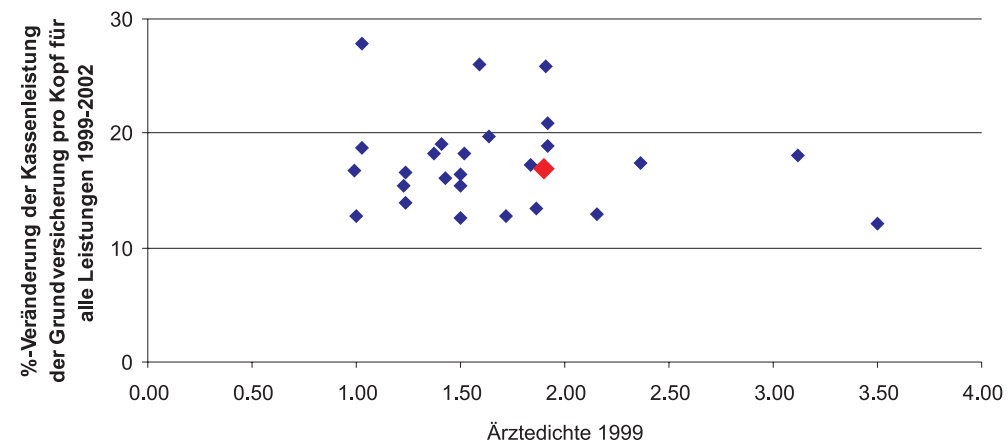

$2 c$

$r=-0.103$

Sig (2-tailed): 0.616

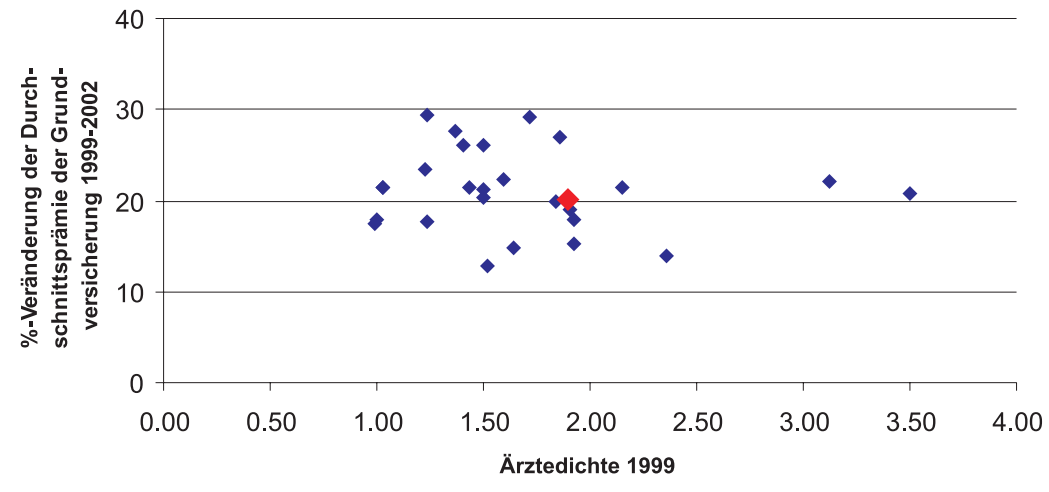

Die einleitend festgehaltene signifikante Korrelation im Querschnitt zwischen der Ärztedichte und den Kassenleistungen bzw. Krankenversicherungsprämien (vgl. Abb. 1) wurde durch Stratifizierung der Daten geprüft. Dabei gruppierten wir die Kantone nach regionalen und strukturellen Merkmalen, um möglichst homogene Schichten zu erhalten.

\section{Ergebnisse}

Die Ärztedichte (Arzt mit Praxistätigkeit pro 1000 Einwohner) betrug 1999 im Mittel 1,90 (Spannweite: 0,99-3,51) und 1,96 (Spannweite: 1,06-3,53) im Jahr 2002. Das entspricht einer Zunahme von 3,16\% innert vier Jahren.

Der Totalaufwand der KVG-Versicherer betrug 1999 Fr. 2014.- und 2002 Fr. 2354.- pro Versicherten $(+16,88 \%)$. Davon beanspruchte die ambulante Behandlung in den Arztpraxen (ohne Medikamente) rund ein Viertel (1999: Fr. 504.-; 2002: Fr. 569.- [+12,9\%]).

Die kantonale Durchschnittsprämie für Erwachsene belief sich 1999 auf Fr. 2448.- pro Jahr und 2002 auf Fr. 2940.- $(+20,1 \%){ }^{\mathrm{a}}$

Die im Querschnitt (Abb. 1) dargestellte hohe positive Korrelation zwischen der momentanen Höhe der Gesundheitsausgaben (und der Versicherungsprämien) und der Ärztedichte erlaubt primär keine Aussagen über einen kausalen $\mathrm{Zu}$ sammenhang.

Im Längsschnitt sind die Korrelationen zwischen der Ärztedichte im Jahr 1999 und den prozentualen Veränderungen der Versicherungsleistungen für die «ambulante Behandlung Arztpraxis» (Abb. 2a) und für die «Kassenleistungen pro Versicherte insgesamt» (Abb. 2b) nicht signifikant (Signifikanzniveau 99,9\%).

Die Korrelation zwischen der Ärztedichte 1999 und der Veränderung der Grundversicherungsprämien in den 26 Kantonen (Abb. 2c) ist ebenfalls nicht signifikant (Signifikanzniveau 99,9\%)

Zwischen den prozentualen Veränderungen der Ärztedichten und den prozentualen Veränderungen der Kassenleistungen für die «ambulante Behandlung in Arztpraxen» (Abb. 3a) bzw. «pro Versicherte insgesamt» (Abb. 3b) besteht statistisch kein signifikanter Zusammenhang. Dieselbe Aussage gilt auch bezüglich Veränderung der Ärztedichte und dem Anstieg der Grundversicherungsprämien pro Versicherte (Abb. 3c).

Durch eine geeignete Stratifizierung der Daten lässt sich der in der Einleitung aufgezeigte signifikante Zusammenhang im Querschnitt zwischen der Ärztedichte und den Kassenlei-

Als Basis wurden die Prämien fü Erwachsene (ab Alter 26 Jahre) inklusive Unfalldeckung bei ordentlicher Grundfranchise von Fr. 230.- angenommen [5]. 
Abbildung $3 \mathrm{a}-\mathrm{c}$

Veränderung der Kassenleistungen und Versicherungsprämien 1999-2002 in Beziehung zur Veränderung der Ärztedichten in 26 Kantonen 1999-2002.

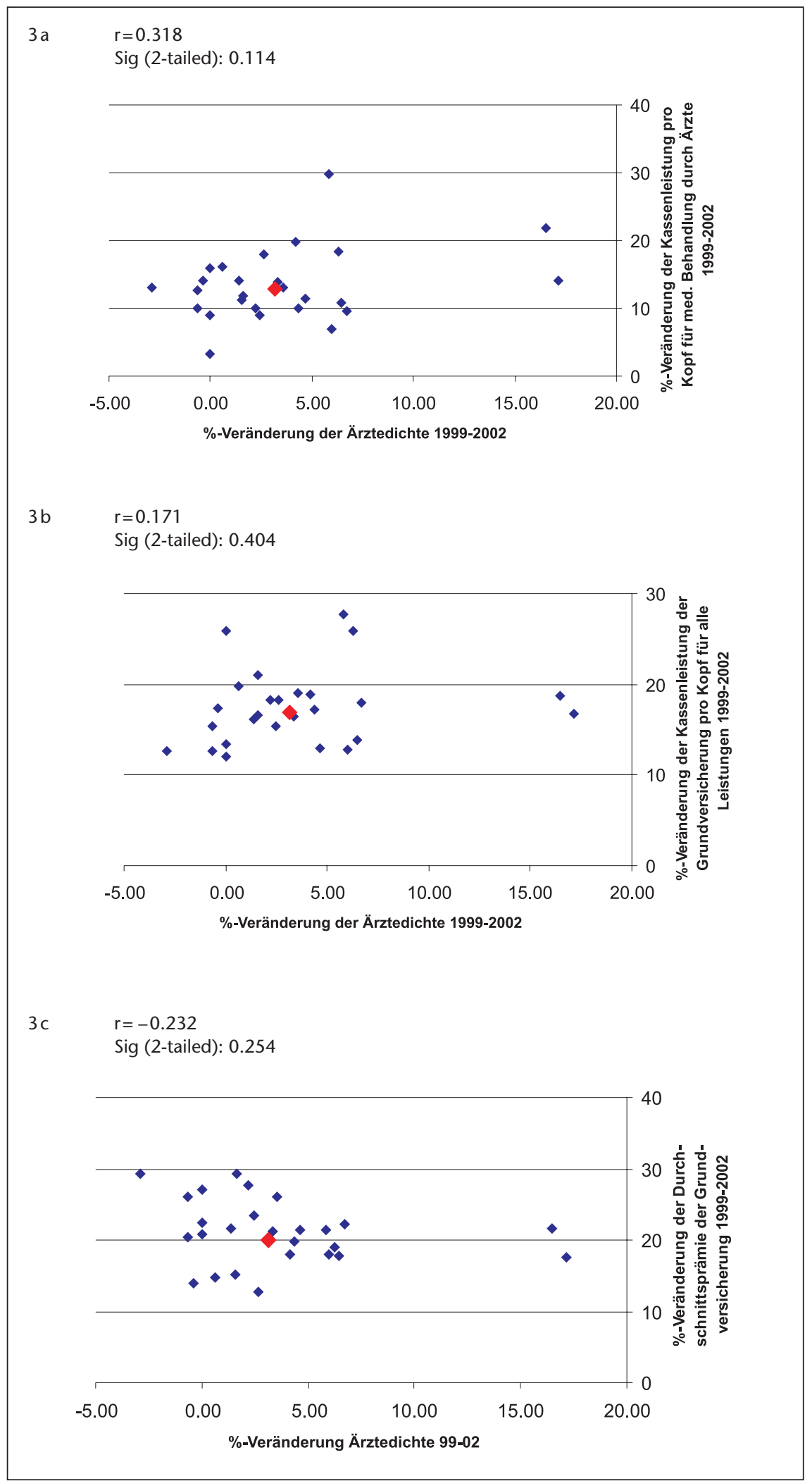

b $\mathrm{N}=12$ : ZG, LU, UR, GL, SZ, NW, OW, AI, AR, SG, TG, GR. stungen bzw. den Krankenversicherungsprämien dann auch widerlegen. Betrachtet man z. B. die ländlichen Kantone der Ost- und Zentralschweiz $^{\mathrm{b}}$, so ist die Korrelation zwischen der Ärztedichte und den Kassenleistungen bzw. den Prämien nicht mehr signifikant (Abb. 4).

Bei den übrigen Kantonen ist weiterhin eine signifikante Korrelation festzustellen. Wenn die Kantone BS, ZH, VD und GE jedoch ausgeklammert werden, ist der Zusammenhang zwischen der Ärztedichte und den Kassenleistungen bzw. den Krankenversicherungsprämien nicht mehr signifikant (Abb. 5). Es scheint somit ein zusätzlicher «Stadt/Land»-Effekt zu existieren, der auf der vorhandenen (kantonalen) Datenbasis nicht sauber filtriert werden kann.

Gesamtschweizerisch stiegen die Durchschnittsprämien für Erwachsene ab Alter 26 Jahre in der obligatorischen Krankenpflegeversicherung von 1999 bis 2002 um 20,1\%. Im ganzen zeigten die Prämiensteigerungen in den 26 Kantonen einen parallelen Verlauf unabhängig von der Ärztedichte (Abb. 6). Dargestellt sind die Verläufe der Prämiensteigerungen für 6 Kantone mit hoher Ärztedichte (BS, GE, VD, ZH, NE, BE) und für 6 Kantone mit niedriger Ärztedichte (GL, TG, SZ, NW, AI, OW). Die Prämien in den Kantonen mit niedrigerer Ärztedichte stiegen sogar etwas stärker als in den Kantonen mit höherer Ärztedichte $(+22,06 \%$ gegenüber $+19,23 \%)$.

\section{Diskussion}

Die Aussage, dass eine zu hohe Ärztedichte die Leistungen der Krankenkassen oder die Prämien in die Höhe getrieben hätte, lässt sich - gestützt auf das analysierte Datenmaterial - statistisch nicht belegen. Veränderungen der Prämien und der Kassenleistungen lassen sich kaum über die Höhe bzw. die Veränderung der Ärztedichte steuern.

Der rein angebotsorientierte Ansatz lässt ausser acht, dass es sich bei der Kostenentwicklung im Gesundheitswesen auch um ein Strukturphänomen handelt. Seit über 100 Jahren weist die Ärztedichte in der Schweiz ein West-Ost-Gefälle auf, das deutlich sozial, weltanschaulich und wirtschaftlich bestimmt ist [15]. Schon 1890 waren die Ärztedichten in der Westschweiz und in den Stadtkantonen Basel und Genf über dreimal höher als in den ländlichen Kantonen der Ost- und Zentralschweiz.

Unsere Analyse stützt sich auf Angaben der Versicherer, deren Qualität wir nicht überprüfen konnten. Beim santésuisse-Datenpool betrug die Abdeckung gesamtschweizerisch 1999 92,8\% und 2002 94,1\%, die Beträge für die Leistungen 
Abbildung 4

Ärztedichte und Kassenleistungen sowie Krankenversicherungsprämien in den ländlichen Kantonen der Ost- und Zentralschweiz.

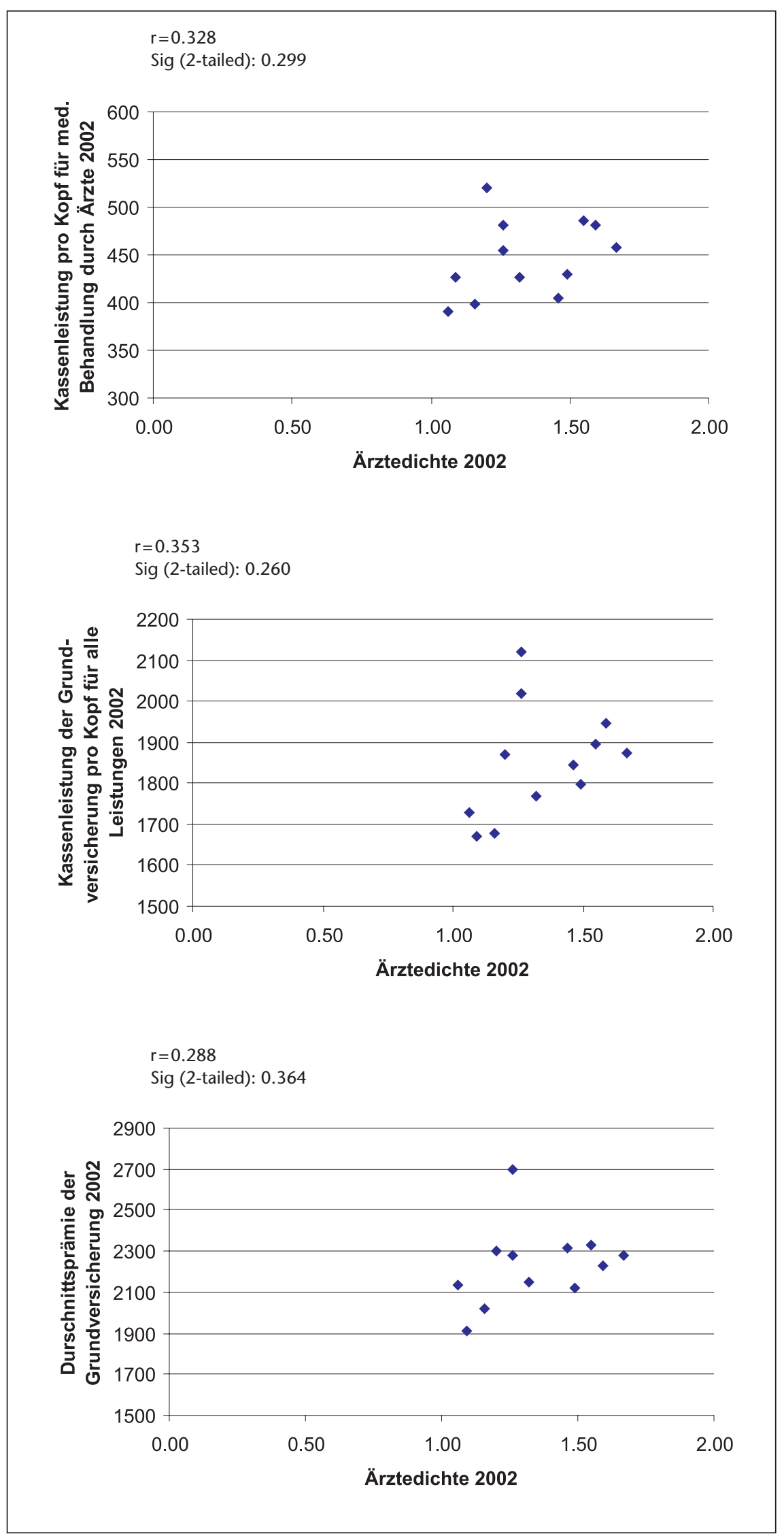

wurden mit Bestandesdaten des Risikoausgleichs auf 100\% hochgerechnet. Bei der Rechnungsstellung durch die Ärzte sind Verschiebungen beim Zeitpunkt der Rechnungsstellung möglich, so dass in einem Jahr durch Verlagerung zu tiefe Leistungen resultieren, im nächsten dann zu hohe.

Die aggregierten Kostendaten erlaubten keine Unterteilung nach der Fachrichtung, so dass die unterschiedlichen Ärztedichten in den Kantonen gleichwertig behandelt werden mussten, ohne Rücksicht auf eine heterogene Zusammensetzung (Facharztmix). Des weiteren stehen die Daten nur nach Kantonen zur Verfügung. Eine Untersuchung der Kostenentwicklung nach anderen Kriterien - z. B. Land/Stadt - ist mittels dieser Daten nicht möglich.

Die Ergebnisse der Studie können aus den genannten Gründen nur als vorläufig betrachtet werden. Weitere Untersuchungen dazu wären notwendig und von Interesse. Hierzu wird es unumgänglich sein, neben der Fortsetzung der vorgelegten Zahlenreihen (und deren Analysen) auch vertiefte Studien über nachfrageorientierte Parameter durchzuführen, entweder in Form von Tracerstudien (mit Fokus auf «teure Krankheiten» und «chronisch Kranke») oder mit Hilfe von geeigneten dynamischen Modellen [16].

\section{Zusammenfassung}

Die vorliegende Untersuchung prüfte die Abhängigkeit der Krankenversicherungsleistungen von der Ärztedichte in 26 Kantonen für die Zeit von 1999 bis 2002. Anhand der santésuisse-Versichertenstatistik, der Sozialversicherungsstatistik des BSV und der FMH-Ärztestatistik wurden die Zusammenhänge wissenschaftlich untersucht. Dabei resultierten folgende Ergebnisse:

Im Querschnitt sind die Leistungsvariablen «ambulante Behandlung Arztpraxis» und «Gesamtleistungen der Krankenversicherung» in den Jahren 1999 und 2002 signifikant mit der Anzahl Ärzte in der freien Praxis korreliert.

Durch eine geeignete Stratifizierung lässt sich der Zusammenhang zwischen den Leistungsvariablen «ambulante Behandlung Arztpraxis» und «Gesamtleistungen der Krankenversicherung» widerlegen.

Im Längsschnitt (1999-2002) sind die Korrelationen zwischen den Ärztedichten in den verschiedenen Kantonen und den prozentualen Veränderungen der Kassenleistungen für die «ambulante Behandlung Arztpraxis» sowie insgesamt pro Versicherten nicht signifikant. 
Abbildung 5

Ärztedichte und Kassenleistungen sowie Krankenversicherungsprämien in den übrigen Kantonen.

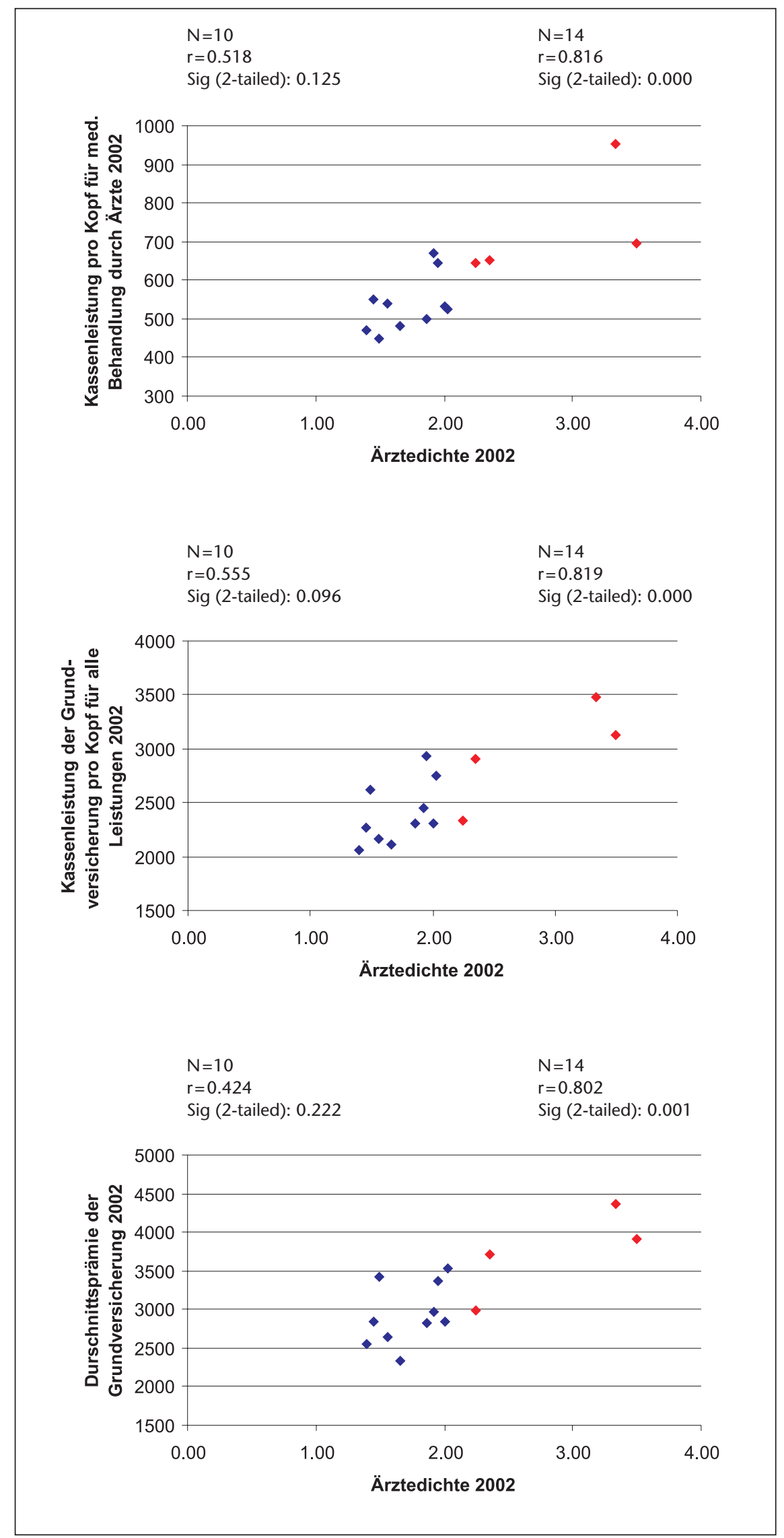

Zwischen dem Anstieg der Grundversicherungsprämien von 1999 bis 2002 und den Ärztedichten 1999 oder mit deren Veränderungen 1999-2002 besteht statistisch kein signifikanter Zusammenhang.

Die Aussage, dass eine zu hohe Ärztedichte die Leistungen der Krankenversicherer oder die Prämien 1999-2002 in die Höhe getrieben habe, lässt sich nicht belegen.

\section{Literatur}

1 Bundesamt für Statistik. Entwicklung der Gesundheitskosten 1960-2000. StatSanté 1/2003.

2 OECD Health DATA 2002 (4th ed): Total expenditure on health-per-capita, US\$ PPP. www.oecd.org.

3 Bundesamt für Statistik. Finanzströme im schweizerischen Gesundheitswesen. StatSanté 2/2002.

4 Definitive santésuisse-Versichertenstatistik 2002 (enthält die Bruttoleistungen absolut und pro Kopf 1999-2002, nach Kantonen). SantésuisseDatenpool, Datenabzug 24. April 2003.

5 Bundesamt für Sozialversicherung (Herausgeber). Schweizerische Sozialversicherungsstatistik 2002, Kapitel Krankenversicherung. Bern: 2002.

6 Verordnung über die Einschränkung der Zulassung von Leistungserbringern zur Tätigkeit zu Lasten der obligatorischen Krankenpflegeversicherung vom 3. Juli 2002 (mit Anhang).

7 BSV-Medienmitteilung vom 3. Juli 2002. www.bsv.admin.ch/aktuell/presse/2002.

8 Scheil-Adlung X. Policies of control of health expenditure through incentives: a comparative analysis on the situation in selected OECD countries. www.issa.int/pdf/jeru98/theme2/2-5a.pdf.

9 Luft HS, Arno P. Impact of increasing physician supply: a scenario for the future. Health Affairs 1986;5(4):31-46.

10 De Jaegher K, Jegers M. A model of physician behaviour with demand inducement. J Health Econ 2000;19:231-58.

11 Burström-Zettermann K, Gisin R. Price setting for doctors. In: Zweifel P, Söderström L (eds.). Regulation for Health: Case studies of Sweden and Switzerland. Dordrecht: Kluwer; 1998. p. 91-109.

12 Heffler S, Smith S, Keehan S, Clemens MK, et al. Health spending projections through 2013. Health Affairs, Web Exclusive, 4 February 2004. www.healthaffairs.org/Archives.

13 FMH. FMH-Ärztestatistik 1999. Schweiz Ärztezeitung 2000;81(16):816-33.

14 FMH. FMH-Ärztestatistik 2002. Schweiz Ärztezeitung 2003;84(17):802-21.

15 Bundesamt für Statistik, Gesundheit. 150 Jahre Bundesstaat. Ärztedichte nach Kantonen. www.bfs.admin.ch/stat_ch/

16 Nocera S, Wanzenried G. On the dynamics of physician density. Theory and empirical evidence for Switzerland. Working Paper No 02.08, Department of Economics, University of Berne. June 2002. www.vwi.unibe.ch/publikationen/download/dp0208.pdf. 
Abbildung 6

Versicherungsleistungen in ausgewählten 12 Kantonen.

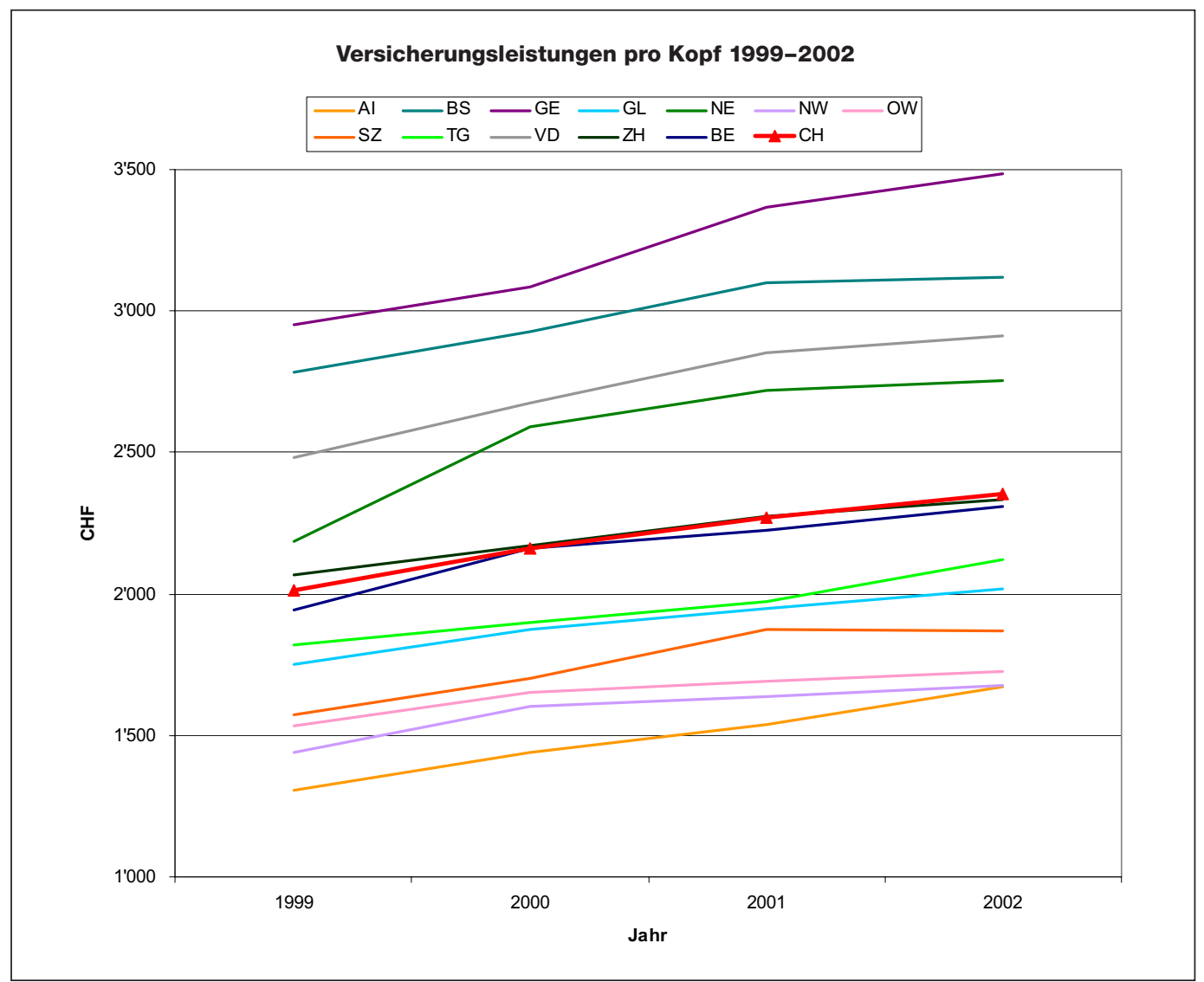

